\title{
Spexin-expressing neurons in the magnocellular nuclei of the human hypothalamus
}

\author{
Artur Pałasz ${ }^{1}$, Aleksandra Suszka-Świtek ${ }^{1}$, Andrzej Kaśkosz² ${ }^{2}$ Danuta Plewka ${ }^{3}$, \\ Katarzyna Bogus ${ }^{1}$, Łukasz Filipczyk ${ }^{1}$, Iwona Błaszczyk ${ }^{1}$, Flora Bacopoulou ${ }^{4}$, \\ John J. Worthington ${ }^{5}$, Aneta Piwowarczyk-Nowak ${ }^{2}$, Marta Tyszkiewicz-Nwafor ${ }^{6}$, \\ Ryszard Wiaderkiewicz ${ }^{1}$
}

${ }^{1}$ Department of Histology, School of Medical Sciences in Katowice, Medical University of Silesia, ul. Medyków 18, 40-752, Katowice, Poland

${ }^{2}$ Department of Anatomy, School of Medical Sciences in Katowice, Medical University of Silesia, ul. Medyków 18, 40-752, Katowice, Poland

${ }^{3}$ Department of Cytophysiology, School of Medical Sciences in Katowice, Medical University of Silesia, ul. Medyków 18, 40-752, Katowice, Poland

${ }^{4}$ Center for Adolescent Medicine and UNESCO Chair on Adolescent Health Care, First Department of Pediatrics, School of Medicine, National and Kapodistrian University of Athens, 'Aghia Sophia' Children's Hospital, Athens, Greece

${ }^{5}$ Division of Biomedical and Life Sciences, Faculty of Health and Medicine, Lancaster University, Lancaster, LA1 4YQ, UK

${ }^{6}$ Department of Child and Adolescent Psychiatry, Poznan University of Medical Sciences, ul. Szpitalna 27/33, 60-572, Poznań, Poland

Corresponding author:

Artur Pałasz

Medyków Street 18, 40-752 Katowice, Poland

apalasz@sum.edu.pl

+48322088377 
Abstract

Neuropeptides are involved in numerous brain activities being responsible for a wide spectrum of higher mental functions. The purpose of this concise, structural and qualitative investigation was to map the possible immunoreactivity of the novel neuropeptide spexin (SPX) within the human magnocellular hypothalamus. SPX is a newly identified peptide, a natural ligand for the galanin receptors (GALR) 2/3, with no molecular structure similarities to currently known regulatory factors. SPX seems to have multiple physiological functions, with an involvement in reproduction and foodintake regulation recently revealed in animal studies. For the first time we describe SPX expressing neurons in the supraoptic (SON) and paraventricular (PVN) nuclei of the human hypothalamus using immunohistochemical and fluorescent methods, key regions involved in the mechanisms of osmotic homeostasis, energy expenditure, consummatory behaviour, reproductive processes, social recognition and stress responses. The vast majority of neurons located in both examined neurosecretory nuclei show abundant SPX expression and this may indirectly implicate a potential contribution of SPX signaling to the hypothalamic physiology in the human brain.

Key words;

spexin, hypothalamus, brain, neuropeptides 


\section{Introduction}

The hypothalamus is a unique brain structure that plays a fundamental role in the origin and integration of central autonomous functions. This includes precise regulation of food intake, osmotic homeostasis, circadian rhythm, reproductive processes, thermoregulation, cardiovascular physiology and even aspects of affective activities. The potential role of hypothalamic related pathways in the origin of several neuropsychiatric dysfunctions is also widely postulated.

Spexin (SPX) is a newly discovered multifunctional neuropeptide acting at both central and peripheral levels. SPX was identified in 2007 by Mirabeau and colleagues (2007) as a transcript of the Ch12orf39 gene. In the rat brain, SPX-expressing neurons have been detected with the highest expression being in the hypothalamic magnocellular nuclei (Porzionato et al. 2010). The chemical structure of SPX is distinctly conserved among species, the rat molecule differs from the human form by only one C-terminal amino acid (Porzionato et al. 2010). SPX is an alternative endogenous ligand for the GALR2/3 receptors, with even higher affinity toward GALR3 than galanin itself (Kim et al. 2014). SPX has recently been linked to multiple physiological functions such as reproduction, food-intake regulation (Ma et al. 2018, Wong et al. 2013), cardiovascular/renal function and nociception (Toll et al. 2012, Porzionato et al. 2012). Due to potent anorexigenic properties of SPX, it has been suggested that a potential excess in hypothalamic SPX signaling may be involved in the pathogenesis of anorexia nervosa (Pałasz et al. 2018). A recent study therefore suggests a possible application of SPX for obesity therapy (Walewski et al. 2014).rSupporting this, glucose-triggered insulin secretion may increase SPX gene expression in the goldfish brain (Ma et al. 2017). A role of SPX as a potential biomarker of glucose metabolism in humans has been also suggested (Hodges et al. 2017). Despite the accumulating animal studies on SPX biology, its distribution and physiology are so far lacking within the human brain. To date, there is no information available about spexin chemoarchitecture in the human hypothalamus. In the present study we aim to provide a structural investigation of the human hypothalamus to reveal the first outline for the neurochemical map of SPX expression within the neurosecretory 
magnocellular nuclei, allowing a potential deeper mechanistic understanding of SPX biology to aid future therapeutic study.

\section{Materials and Methods}

\subsection{Immunohistochemistry}

Human brain tissue specimens with no neuropathological findings were obtained from the Conscious Body Donation Program conducted by the Department of Anatomy at the Medical University of Silesia in Katowice. Brains were post mortem perfusion-fixed with $4 \%$ paraformaldehyde buffered solution $(\mathrm{pH}$ 7.2-7.4) and then immersion-fixed over a period of at least three months. The rostral hypothalamus was precisely excised from 2 diencephalic slices ( $n=2)$ according to the referenced human brain atlases (Mai et al. 2015, Cho 2014) as visualized in Figure 1. Samples were dehydrated, embedded in paraffin wax and finally sectioned by microtome (Leica Microsystems, Germany) at $10 \mu \mathrm{m}$ thick serial slices. The inferior hypothalamic boundary was identified in the coronal plane by the optic chiasm and superior boundary was delineated by the anterior commissure. Third ventricle and edge of ventral pallidum (parallel to diagonal band of Broca) formed lateral and medial borderlines respectively. The sections were deparaffinized with xylene and rehydrated to $50 \%$ ethanol via graduated alcohol solutions.

After rehydration and subsequent antigen retrieval with citrate buffer $(\mathrm{pH} 4.0)$ solution (Vector Laboratories) at $60{ }^{\circ} \mathrm{C}$ sections were rinsed three times for $5 \mathrm{~min}$ in 0.05 M TBS-saline ( $\mathrm{pH} 7.6$ ) and placed in PBS with $0.1 \%$ Triton X-100 (Sigma). They were blocked with $10 \%$, goat serum and incubated overnight at $4^{\circ} \mathrm{C}$ with rabbit anti-rat spexin polyclonal antiserum (1:2000, Phoenix Pharmaceuticals). Additionally, for a complementary internal reference some specimens were placed in goat monoclonal antibody against rat nesfatin-1 (1:1000, Enzo Life Sciences). All sections were treated with TrueBlack $^{\circledast}$ (Biotium, Hayward, CA, USA) to remove unwanted lipofuscin autofluorescence. Following incubation with primary antibodies brain sections were kept in complete darkness with secondary antibodies labeled with FITC or TRITC (1:200, Abcam) for SPX, nesfatin-1 respectively, and mounted on slides with DAPIcontaining medium. Alternatively, rabbit anti-rat spexin polyclonal antibody (1:500 Phoenix Pharmaceuticals) was followed by biotinylated goat anti-rabbit secondary antibody and then an avidin-biotin-horseradish peroxidase complex (Vectastain ABC 
kit, Vector Labs) and visualised with3,3'-diaminobenzidine (DAB). Because rodent and human SPX molecules differ in only one amino acid we checked the SPX antibody specificity with an absorption test with human SPX (Phoenix Pharmaceuticals) as previously reported (Gu et al. 2015). The crucial point of this test was a preincubation of the SPX (pure antigen) with the antibody before the essential IHC reaction. Moreover, this antiserum has also been verified using series of human positive controls (stomach and intestine tissues). All sections were mounted on glass slides, dehydrated and coverslipped. For basic neurostructural evaluation, representative sections were stained via Nissl method in $1 \%$ Cresyl violet for 60 mins. After rinsing and differentiation by acetic acid and mounting with DPX, sections were cover slipped. All images were captured with Nikon Coolpix fluorescent optic systems and processed using Image ProPlus software (Media Cybernetics, USA). The SON/PVN cyto- and chemoarchitecture was analyzed and immunopositive cells were counted using Image J 1.43u software. The anatomical coordinates and structure of the hypothalamus were defined after Borgers et al. (2013). The number of SPX-positive cells was counted in SON and PVN nuclei to obtain the density of these cells per standardized area $\left(0.16 \mathrm{~mm}^{2}\right.$, frame $\left.400 \times 400 \mu \mathrm{m}\right)$. Data are presented as a mean \pm standard error of the mean (SEM).

\section{Results}

Upon examining the SON, a distinct majority of large-sized, oval or round neurons showed intense SPX immunostaining and fluorescence (Fig.1 and 2). Other, smaller cells that displayed a wide spectrum of shapes (fusiform, droplet-shaped or multipolar perikarya) exhibited medium SPX immunoreactivity (Fig.2). The highest density of large, SPX-positive cells were observed in the posterior SON sections; mean number of SPX-positive cells in SON ranged from $10 \pm 2 \%$ in the rostral to $37 \pm 2 \%$ in the caudal part of the nucleus (Fig.2). In the SON a bigger proportion of multiform neuropeptide immunopositive cells was found which, depending on section plane, presented a more anterior orientation. Of note, the medium-sized, oval-shaped perikarya of the entire SON exhibit a distinct but not intense SPX immunoreactivity (Fig.2; B1-B3). Most SPX-expressing neurons in the PVN had large elongated, irregular-shaped, multipolar or triangular perikarya but smaller round cells were also 
found (Fig. 2; B,D,E). The intensity of fluorescence was slightly lower when compared to the SON. Mean number of SPX-positive cells in the PVN ranged from $17 \pm 2 \%$ in the rostral, to $29 \pm 2 \%$ in the caudal part of the nucleus. (Fig.2). Looking at the intracellular aspect of SPX immunohistochemistry regular reticular and perinuclear DAB staining indicates a labelling of the endoplasmic reticulum and Golgi apparatus,especially within the large SON neurons (Fig 2.). Within some of these cells, aslightly thickened nuclear membrane was distinctly DAB stained. The results of the fluorescence staining was also evenly distributed in the SON neurons, while some SPX immunopositive granules and varicosities were also identified in several long axons located between the SON neurosecretory cells. Overall immunoreactivity was less abundant in the PVN neurons, however those with oval morphology had exposed dense reticular staining of both the perinuclear and peripheral cell region; while axonal positivity in the PVN was poorly marked or even absent.

\section{Discussion}

We have demonstrated for the first time SPX immunoreactive neurons in the human magnocellular hypothalamus, suggesting that this novel neuropeptide may be involved in autonomic functions mediated by this brain region. The chemoarchitecture of SPX neurons in the SON and PVN is similar to the distribution of nesfatin-1 which previously was shown to colocalize with oxytocin and vasopressin (Psilopanagioti et al. 2019), CRH (Bao and Swaab 2010), 26RFa (Bruzzone et al. 2006), CART (Elias et al. 2001) and galanin (Gai et al. 1990). SPX immunoreactivity was the most abundant in the large round, fusiform and multipolar perikarya that represent type II and III human SON neurons, as defined by the Golgi method-based morphological classification (AIHussain and Al-Jomard 1996). Most positive cells were bipolar with short and simple branching dendritic processes (Armstrong 1995). Of note, the size of SON/PVN neurons may enlarge with normal aging (Vogel et al. 1990). The majority of smaller, elongated and ovoidal type I and IV somata were characterized by lower intensity of SPX-immunoexpression. The most prominent SPX-expression was observed in the vasopressinergic magnocellular part of PVN occupying ventral area of the nucleus. $A$ dorsally located population of large spindle-shaped perikarya was also SPX-positive. Previous studies revealed that this group of human PVN neurons have a distinct tyrosin 
hydroxylase expression and release oxytocin (Qin et al. 2018, Koutcherov et al. 2000). The present study revealed that the SPX expression in the human SON and PVN is homologous to the neuropeptide chemoarchitecture in the rat magnocellular nuclei (Porzionato et al. 2010), adding weight to the translational relevance of previous rat studies. Given a significant co-distribution of SPX, vasopressin and oxytocin in cells within the hypothalamus, this may potentially indicate functional interactions between these neuropeptides, but this should be cautiously taken into consideration. SPX may therefore potentially be involved in the central control of osmotic homeostasis and reproductive processes in humans. Since SON and PVN neurons receive both excitatory and inhibitory afferents from other hypothalamic, limbic and brainstem structures, (lovino et al. 2016, Leng et al. 1999) it should not be excluded that SPX synthesis may be regulated by these brain centres.

At present, there is no clear consensus on the contribution of SPX to human hypothalamus function, moreover it is not yet known how SPX and its receptors are regulated in this central autonomic structure, crucial for homeostatic balance. The pattern of SPX distribution in the human hypothalamus mirrors the rat (Porzionato et al. 2010) and to some extent even goldfish (Wong et al. 2013), with the most intense immunoreactivity in SON and slightly lower in PVN. One can therefore suggest that SPX role in the human hypothalamic nuclei may be potentially analogous to that revealed in the animal brain. A presence of SPX in the magnocellular neurosecretory centres suggests that this regulatory factor may be secreted into the blood via the hypophyseal circulation and can act as a multifunctional neurohormone.

Animal studies, have shown that SPX reduces food intake and body weight and may induce satiety via blockage of neuropeptide Y/orexin signalling (Ma et al. 2015) and by suppressing AgRP (Zheng et al. 2017). Thus, SPX is currently considered as a potent central, and probably peripheral, novel anorexigenic neuropeptide. SPX can also increase locomotion and lipid oxidation and improve glucose tolerance (Walewski et al. 2014). Endogenous SPX acting as a ligand of GalR2 and GalR3 receptors could also play a role in the pathophysiology of depression and anxiety (Swanson et al. 2005).

GalR2 shows a high distribution in several hypothalamic nuclei (Bartfai et al. 1993) including SON and PVN (Gundlach and Burazin 1998), where they can potentially be stimulated by SPX. Hence, a variety of biological effects exerted by central galanin signalling such as regulation of energy homeostasis (feeding, 
metabolism and body weight control), cognitive functions, learning and memory (Bartfai et al. 1992,1993) may be SPX-related. At the level of hypothalamic circuits, galanin stimulates feeding behavior (Leibowitz et al. 1992), growth hormone (Bauer et al. 1986, Murakami et al. 1987) and prolactin (Koshiyama et al. 1990) exocytosis, but inhibits corticosterone release (Tempel et al. 1990). The central anti-nociceptive actions of galanin (Xu et al. 2010) are exerted via GalR1 (Fu et al. 2011) and GalR2 activation (Zhang et al. 2017) in the hypothalamic nuclei (Amorim et al. 2015, Gu et al. 2007), nucleus accumbens (Duan et al. 2015), central nucleus of amygdala (Jin et al. 2010) or in the spinal cord (Xu et al. 2012). This important effect of GalR2 activation may be also related to local SPX release within the hypothalamus, that may modulate nociceptive signalling mechanisms (Toll et all. 2012).

The latest study reveals that SPX may participate in serotonin-dependent regulation of anxiety behaviour in zebrafish (Jeong et al. 2019). It was recently reported that long-term treatment with selective serotonin reuptake inhibitors escitalopram decreased the SPX expression in the rat hypothalamus but increased levels in the hippocampus and striatum (Pałasz et al. 2016). Studies on primary cultures of mouse immortalized hypothalamic neurons showed that SPX mRNA expression is acutely modulated by palmitate and nitric oxide. Stress-related injury of endoplasmic reticulum as well as C/EBP-b signalling also affect both SPX and GALR2/3 gene expression in these cells (Tran et al. 2019). SPX is also involved in the hypothalamus-related control of cardiovascular physiology and reproductive processes (Porzionato et al. 2012, Liu et al. 2019, Kumar et al. 2018, Lv et al. 2018). Of note, a recent study reported increased serum SPX concentrations in pregnant women with gestational diabetes mellitus (Akbas et al. 2019). Nothing is known about the factors regulating SPX expression in either animal or human brains, therefore a large spectrum of speculative possibilities, including the aforementioned should be taken into consideration.

Given the small number of brain samples, it is worth considering expanding the study to complement our initial report. Nevertheless, given the scarcity of human brain tissue, we believe that this initial report will offer a much-needed initial neurochemical map of SPX expression. Although confirmation of SPX neurochemistry requires numerous further neurostructural and molecular studies, even at the present stage of knowledge this regulatory peptide can be considered as an interesting and potentially important regulatory factor in the human hypothalamus. 
At present, a role of SPX in human neurophysiology still remains an area of speculation, but undoubtedly further studies on this regulatory factor, e.g. on its coexpression with other important neuropeptides both classical (vasopressin, oxytocin, CRF, NPY, somatostatin) and novel (nesfatin-1, phoenixin, alarin) definitely merits attention. Moreover, the 3-D visualization of the aforementioned neuropeptide distribution in the whole human hypothalamus, as well as study of potential interactions between these factors is of paramount importance. To conclude, our investigation presents the novel identification of SPX expressing neurons in the human hypothalamus and their assemblies show similar patterns of distribution in SON and PVN. The study demonstrates for the first time a presence of SPX in the human brain which could implicate a potential contribution of this neuropeptide to numerous central neurosecretory mechanisms.

\section{Conclusions}

To conclude, our investigation presents the novel identification of SPX expressing neurons in the human hypothalamus and their assemblies show similar patterns of distribution in SON and PVN. The study demonstrates for the first time a presence of SPX in the human brain which could implicate a potential contribution of this neuropeptide to numerous central neurosecretory mechanisms.

\section{Acknowledgements}

The authors would like to thank Prof. Marcin Ruciński, PhD, DSc from Poznań University of Medical Sciences and Mr Paweł A. Kołodziejski, MSc from Poznań University of Life Sciences for their valuable technical assistance.

\section{CRediT authorship contribution statement}


A. Pałasz: Conceptualization, Investigation, Data curation, Writing - original draft. A. Suszka-Świtek, A. Kaśkosz, D. Plewka, K. Bogus, L. Filipczyk, I. Błaszczyk: Methodology, Immunohistochemistry, Tissue aquisition, F. Bacopoulou, A. Piwowarczyk-Nowak: Resources. M.Tyszkiewicz-Nwafor, J.J.Worthington: Formal analysis. R. Wiaderkiewicz: Project administration.

\section{Declaration of Competing Interest}

The authors declare that they have no known competing interests.

\section{References}

Akbas, M., Koyuncu, F.M., Oludag Mete, T., Taneli, F., Ozdemir, H., Yilmaz, O., 2019. Serum levels of spexin are increased in the third trimester pregnancy with gestational diabetes mellitus. Gynecol. Endocrinol. 35, 1050-1053.

Al-Daghri, N.M., Wani, K., Yakout, S.M., Al-Hazmi, H., Amer, O.E., Hussain, S.D., Sabico, S., Ansari, M.G.A., Al-Musharaf, S., Alenad, A.M., Alokail, M.S., Clerici, M., 2019. Favorable Changes in Fasting Glucose in a 6-month Self-Monitored Lifestyle Modification Programme Inversely Affects Spexin Levels in Females with Prediabetes. Sci. Rep. 9, 9454.

Al-Hussain, S., Al-Jomard, R., 1996. Morphology of neurons in the anterior hypothalamic area and supraoptic hypothalamic nucleus of the adult human brain. Ital. J. Neurol. Sci. 17, 261-266.

Amorim, D., Viisanen, H., Wei, H., Almeida, A., Pertovaara, A., Pinto-Ribeiro, F., 2015. Galanin-mediated behavioural hyperalgesia from the dorsomedial nucleus of the hypothalamus involves two independent descending pronociceptive pathways. PLoS One 10, e0142919.

Armstrong, W.E., 1995. Morphological and electrophysiological classification of hypothalamic supraoptic neurons. Prog. Neurobiol. 47, 291-339.

Bartfai, T., Fisone, G., Langel, U., 1992. Galanin and galanin antagonists: molecular and biochemical perspectives. Trends Pharmacol. Sci. 13, 312-317.

Bartfai, T., Hökfelt, T., Langel, U., 1993. Galanin--a neuroendocrine peptide. Crit. Rev. Neurobiol 7, 229-274. 
Bauer, F.E., Ginsberg, L., Venetikou, M., MacKay, D.J., Burrin, J.M., Bloom, S.R., 1986. Growth hormone release in man induced by galanin, a new hypothalamic peptide. Lancet 2, 192-194.

Bao, A.M., Swaab, D.F., 2010. Corticotropin-releasing hormone and arginine vasopressin in depression focus on the human postmortem hypothalamus. Vitam. Horm. 82, 339-365.

Borgers, A.J., Alkemade, A., Van de Giessen, E.M., Drent, M.L., Booij, J., Bisschop, P.H., Fliers, E., 2013. Imaging of serotonin transporters with [123I]FP-CIT SPECT in the human hypothalamus. EJNMMI Res. 25, 34.

Bruzzone, F., Lectez, B., Tollemer, H., Leprince, J., Dujardin, C., Rachidi, W., Chatenet, D., Baroncini, M., Beauvillain, J.C., Vallarino, M., Vaudry, H., Chartrel, N., 2006. Anatomical distribution and biochemical characterization of the novel RFamide peptide 26RFa in the human hypothalamus and spinal cord. J. Neurochem. 99, 616627.

Cho, Z.-H., 2014. 7.0 Tesla MRI Brain Atlas: In-vivo atlas with cryomacrotome correlation. Springer, Berlin

Duan, H., Zhang, Y., Zhang, X.M., Xu, H.H., Shu, J., Xu, S.L., 2015. Antinociceptive roles of galanin receptor 1 in nucleus accumbens of rats in a model of neuropathic pain. J. Neurosci. Res. 93,1542-1551.

Elias, C.F., Lee, C.E., Kelly JF, Ahima, R.S., Kuhar, M., Saper, C.B., Elmquist, J.K., 2001. Characterization of CART neurons in the rat and human hypothalamus. J. Comp. Neurol. 26, 1-19.

Fu, L.B., Wang, X.B., Jiao, S., Wu, X., Yu, L.C., 2011. Antinociceptive effects of intracerebro-ventricular injection of the galanin receptor 1 agonist M 617 in rats. Neurosci. Lett. 491, 174-176.

Gai, W.P, Geffen, L.B., Blessing, W.W., 1990. Galanin immunoreactive neurons in the human hypothalamus: colocalization with vasopressin-containing neurons. J. Comp. Neurol. 298, 265-80.

Gu, L., Ma, Y., Gu, M., Zhang, Y., Yan, S., Li, N., Wang, Y., Ding, X., Yin, J., Fan, N., Peng, $Y .$, 2015. Spexin peptide is expressed in human endocrine and epithelial tissues and reduced after glucose load in type 2 diabetes. Peptides 71, 232-239.

Gu, X.L., Sun, Y.G., Yu, L.C., 2007. Involvement of galanin in nociceptive regulation in the arcuate nucleus of hypothalamus in rats with mononeuropathy. Behav. Brain. Res. 179, 331-335.

Gundlach, A.L., Burazin, T.C., 1998. Galanin-galanin receptor systems in the hypothalamic paraventricular and supraoptic nuclei. Some recent findings and future challenges. Ann. N.Y. Acad. Sci. 863, 241-251. 
Hodges, S.K., Teague, A.M., Dasari, P.S., Short, K.R., 2018. Effect of obesity and type 2 diabetes, and glucose ingestion on circulating spexin concentration in adolescents. Pediatr. Diabetes 19, 212-216.

lovino, M., Giagulli, V.A., Licchelli, B., lovino, E., Guastamacchia, E., Triggiani, V., 2016. Synaptic Inputs of Neural Afferent Pathways to Vasopressin- and OxytocinSecreting Neurons of Supraoptic and Paraventricular Hypothalamic Nuclei. Endocr. Metab. Immune Disord. Drug Targets. 16, 276-287.

Jeong, I., Kim, E., Seong, J.Y., Park, H.C., 2019. Overexpression of Spexin 1 in the Dorsal Habenula Reduces Anxiety in Zebrafish. Front. Neural Circuits 13, 53.

Jin, W.Y., Liu, Z., Liu, D., Yu, L.C., 2010. Antinociceptive effects of galanin in the central nucleus of amygdala of rats, an involvement of opioid receptors. Brain Res. $1320,16-21$.

Koshiyama, H., Shimatsu, A., Kato, Y., Assadian, H., Hattori, N., Ishikawa, Y., Tanoh, T., Yanaihara, N., Imura, H., 1990). Galanin-induced prolactin release in rats: pharmacological evidence for the involvement of alphaadrenergic and opioidergic mechanisms. Brain Res. 507, 321-324.

Koutcherov, Y., Mai, J.K., Ashwell, K.W., Paxinos, G., 2000. Organization of the human paraventricular hypothalamic nucleus. J. Comp. Neurol. 423, 299-318.

Kumar, S., Hossain, M.J., Javed, A., Kullo, I.J., Balagopal, P.B., 2018. Relationship of circulating spexin with markers of cardiovascular disease: a pilot study in adolescents with obesity. Pediatr. Obes. 13, 374-380.

Leibowitz, S.F., 1992. Neurochemical-neuroendocrine systems in the brain controlling macronutrient intake and metabolism. Trends Neurosci. 15, 491-497.

Leng, G., Brown, C.H., Russell, J.A., 1999. Physiological pathways regulating the activity of magnocellular neurosecretory cells. Prog. Neurobiol. 57, 625-655.

Lim, C.H., Lee, M.Y.M., Soga, T., Parhar, I., 2019. Evolution of Structural and Functional Diversity of Spexin in Mammalian and Non-mammalian Vertebrate Species. Front. Endocrinol. (Lausanne) 19, 379.

Liu, Y., Sun, L., Zheng, L., Su, M., Liu, H., Wei, Y., Li, D., Wang, Y., Dai, C., Gong, Y., Zhao, C., Li, Y., 2020. Spexin protects cardiomyocytes from hypoxia-induced metabolic and mitochondrial dysfunction. Naunyn Schmiedebergs Arch. Pharmacol. 393, 25-33.

Lv, S.Y., Zhou, Y.C., Zhang, X.M., Chen, W.D., Wang, Y.D., 2019. Emerging Roles of NPQ/Spexin in Physiology and Pathology. Front. Pharmacol. 10, 457.

Ma, A., Bai, J., He, M., Wong, A.O.L., 2018. Spexin as a neuroendocrine signal with emerging functions. Gen. Comp. Endocrinol. 265, 90-96.

Mai, J., Majtanik, M., Paxinos, G., 2015. Atlas of the human brain. Elsevier, London 
Murakami, Y., Kato, Y., Koshiyama, H., Inoue, T., Yanaihara, N., Imura, H., 1987. Galanin stimulates growth hormone $(\mathrm{GH})$ secretion via $\mathrm{GH}$-releasing factor (GRF) in conscious rats. Eur. J. Pharmacol. 136, 415-418.

Pałasz, A., Janas-Kozik, M., Borrow, A., Arias-Carrión, O., Worthington, J.J., 2018. The potential role of the novel hypothalamic neuropeptides nesfatin-1, phoenixin, spexin and kisspeptin in the pathogenesis of anxiety and anorexia nervosa. Neurochem. Int. 113, 120-136.

Pałasz, A., Suszka-Świtek, A., Filipczyk, Ł., Bogus, K., Rojczyk, E., Worthington, J., Krzystanek, M., Wiaderkiewicz, R., 2016. Escitalopram affects spexin expression in the rat hypothalamus, hippocampus and striatum. Pharmacol. Rep. 68, 1326-1331.

Porzionato, A., Rucinski, M., Macchi, V., Stecco, C., Malendowicz, L.K., De Caro, R., 2010. Spexin expression in normal rat tissues. J Histochem Cytochem. 58: 825-837

Porzionato, A., Rucinski, M., Macchi, V., Stecco, C., Sarasin, G., Sfriso, M.M., Di Giulio, C., Malendowicz, L.K., De Caro, R., 2012. Spexin is expressed in the carotid body and is upregulated by postnatal hyperoxia exposure. Adv. Exp. Med. Biol. 758, 207-213.

Psilopanagioti, A., Nikou, S., Papadaki, H., 2019. Nucleobindin-2/Nesfatin-1 in the Human Hypothalamus Is Reduced in Obese Subjects and Colocalizes with Oxytocin, Vasopressin, Melanin-Concentrating Hormone, and Cocaine- and AmphetamineRegulated Transcript. Neuroendocrinology. 108, 190-200.

Qin, C., Li, J., Tang, K., 2018. The Paraventricular Nucleus of the Hypothalamus: Development, Function, and Human Diseases. Endocrinology. 159, 3458-3472.

Reyes-Alcaraz, A., Lee, Y.N., Son, G.H., Kim, N.H., Kim, D.K., Yun, S., Kim, D.H., Hwang, J.I., Seong, J.Y., 2016. Development of Spexin-based Human Galanin Receptor Type II-Specific Agonists with Increased Stability in Serum and Anxiolytic Effect in Mice. Sci. Rep. 6, 21453.

Sonmez, K., Zaveri, N.T., Kerman, I.A., Burke, S., Neal, C.R., Xie, X., Watson, S.J., Toll, L., 2009. Evolutionary sequence modeling for discovery of peptide hormones.

PLoS Comput. Biol. 5, e1000258.

Swanson, C.J., Blackburn, T.P., Zhang, X., Zheng, K., Xu, Z.Q., Hökfelt, T., Wolinsky, T.D., Konkel, M.J., Chen, H., Zhong, H., Walker, M.W., Craig, D.A., Gerald, C.P., Branchek, T.A., 2005. Anxiolytic- and antidepressant-like profiles of the galanin-3 receptor (Gal3) antagonists SNAP 37889 and SNAP 398299. Proc. Natl. Acad Sci. USA 48,17489-17494.

Tempel, D.L., Leibowitz, S.F.,1990. Galanin inhibits insulin and corticosterone release after injection into the PVN. Brain. Res. 536, 353-357.

Toll, L., Khroyan, T.V., Sonmez, K., Ozawa, A., Lindberg, I., McLaughlin, J.P., Kapusta, D.R., Eans, S., Shahien, A., 2012. Peptides derived from the prohormone 
proNPQ/spexin are potent central modulators of cardiovascular and renal function and nociception. The FASEB Journal, 26, 947-954.

Tran, A., Loganathan, N., Mcllwraith, E.K., Belsham, D.D., 2019. Palmitate and Nitric Oxide Regulate the Expression of Spexin and Galanin Receptors 2 and 3 in Hypothalamic Neurons. Neuroscience S0306-4522(19)30720-1.

Vogels, O.J., Broere, C.A., Nieuwenhuys, R., 1990. Neuronal hypertrophy in the human supraoptic and paraventricular nucleus in aging and Alzheimer's disease. Neurosci. Lett. 109, 62-67.

Walewski, J.L., Ge, F., Lobdell, H. 4th, Levin, N., Schwartz, G.J., Vasselli, J.R., Pomp, A., Dakin, G., Berk, P.D., 2014. Spexin is a novel human peptide that reduces adipocyte uptake of long-chain fatty acids and causes weight loss in rodents with dietinduced obesity. Obesity 22, 1643-1652.

Xu, X., Yang, X., Zhang, P., Chen, X., Liu, H., Li, Z., 2012. Effects of exogenous galanin on neuropathic pain state and change of galanin and its receptors in DRG and SDH after sciatic nerve-pinch injury in rat. PLoS One 7, e37621.

Xu, X.J., Hokfelt, T., Wiesenfeld-Hallin, Z., 2010. Galanin and spinal pain mechanisms: past, present, and future. EXS 102, 39-50.

Zhang, M.L., Wang, H.B., Fu, F.H., Yu, L.C., 2017. Involvement of galanin and galanin receptor 2 in nociceptive modulation in anterior cingulate cortex of normal rats and rats with mononeuropathy. Sci. Rep. 7, 45930.

Zheng, B., Li, S., Liu, Y., Li, Y., Chen, H., Tang, H., Liu, X., Lin, H., Zhang, Y., Cheng, C.H.K., 2017. Spexin Suppress Food Intake in Zebrafish: Evidence from Gene Knockout Study. Sci. Rep. 7, 14643. 


\section{Figure captions}

Fig.1. Macroscopic overview of the serial brain sections containing examined hypothalamic regions, Nissl staining (top panel). Scale bar: $5 \mathrm{~mm}$. Supraoptic, SON and paraventricular, PVN nuclei (yellow); anterior commissure, ac; lateral ventricle, Iv; hypothalamus, hyp; third ventricle, 3v. Microstructure of SON; fig. A, A1 and PVN fig. F, F1. Nissl staining. Scale bars: $200 \mu \mathrm{m}(\mathrm{A}, \mathrm{F})$ and $100 \mu \mathrm{m}(\mathrm{A} 1, \mathrm{~F} 1)$. Spexin (SPX) expression in the magnocellular hypothalamus. Immunohistochemistry (DAB), supraoptic nucleus; fig. B-E, paraventricular nucleus; fig. G-J. Scale bars: $200 \mu \mathrm{m}$. Fluorescence (FITC), SON; fig. B1-E1, PVN; fig. G1-J1. Scale bars: $100 \mu \mathrm{m}$.

Fig.2. Mean number of SPX immunopositive cells per standarized frame $\left(0.16 \mathrm{~mm}^{2}\right)$ counted from rostral to caudal magnocellular SON and PVN (upper panel). A selection of neurons under higher magnification. Immunohistochemical DAB reaction for SPX in SON (fig. A,D), fluorescence for SPX (FITC, fig. B,E) and nesfatin-1 (TRITC; fig. C,F) Scale bars: $50 \mu \mathrm{m}$ (fig. A-D), $20 \mu \mathrm{m}$ (fig. E-F).

\section{Highlights}

- SPX-expressing neurons are present in the human supraoptic and paraventricular nuclei

- SPX immunoreactivity is slightly higher in the supraoptic than in the paraventricular nucleus

- The highest density of SPX-positive neurons is observed in the posterior part of supraoptic nucleus 
